\title{
The origin of dynamic scaling in dilute polymer solutions
}

\author{
P. Sunthat* and J. Ravi Prakash $\dagger$ \\ Department of Chemical Engineering, Monash University, Melbourne, VIC 3800, Australia
}

(Dated: February 8, 2020)

\begin{abstract}
The hydrodynamic radius of a polymer chain, obtained using Brownian dynamics simulations of the continuum Edwards model, is found to obey a crossover in the excluded volume parameter $z$, which is significantly different from that observed for the radius of gyration. It is shown that this difference arises from contributions due to dynamic correlations to the diffusivity, which are ignored in the commonly used definition of hydrodynamic radius based on the Kirkwood expression. The swelling of the hydrodynamic radius from the $\theta$-state, obtained from simulations, shows remarkable agreement with experimental measurements.

PACS numbers: 61.25.Hq, 82.35.-x, 83.10.Mj
\end{abstract}

The mean size of a polymer in a dilute solution can be obtained by static measurements that yield the radius of gyration $R_{\mathrm{g}}$ or by dynamic experiments that provide the diffusion coefficient $D$. An outstanding problem yet unresolved is the observed difference in the scaling with molecular weight between static and dynamic measurements [1]. For instance, in the limit of large molecular weight $M, R_{\mathrm{g}}$ scales as $M^{0.59}$, while the hydrodynamic radius $R_{\mathrm{H}}\left(\propto D^{-1}\right)$ scales as $M^{0.57}$ [2]. In addition, in the crossover regime between the $\theta$-state and the good solvent limit, the growth of $R_{\mathrm{g}}$ and $R_{\mathrm{H}}$ relative to the $\theta$-state, in terms of the scaling variable $z=$ $v_{0}\left(1-T_{\theta} / T\right) \sqrt{M}$, is observed to be significantly different. Here, $v_{0}$ is a chemistry dependent prefactor, $T$ is the temperature, and $T_{\theta}$ is the temperature at the $\theta$-state. Theoretical attempts to explain the origin of this anamalous dynamic scaling behavior have mainly based the definition of $R_{\mathrm{H}}$ on the Kirkwood expression for diffusivity [3, 4, 5, 6]. In this work, we show using exact Brownian Dynamics simulations of the continuum Edwards model [7], that (i) the use of Kirkwood's expression in fact leads to nearly identical scaling in the two cases, (ii) in order to exhibit dynamic scaling that is distinct from static scaling, $R_{\mathrm{H}}$ should be based on the long-time diffusivity, and (iii) the latter definition of $R_{\mathrm{H}}$ leads to excellent agreement with experimental observations.

Theoretical treatments of the static and dynamic properties of polymers in a dilute solution are typically carried out with a bead-spring chain model, which consists of $N$ beads connected by linear springs. The swelling of a polymer in a good solvent is usually represented in terms of the ratio of the size in the presence of excluded volume (EV) interactions between the beads to size in the $\theta$-state. The swelling of the radius of gyration is denoted as, $\alpha_{\mathrm{g}}=R_{\mathrm{g}} / R_{\mathrm{g}}^{\theta}$ and that of the hydrodynamic radius as $\alpha_{\mathrm{H}}=R_{\mathrm{H}} / R_{\mathrm{H}}^{\theta}$. The hydrodynamic radius $R_{\mathrm{H}}$ is often approximated by a static measure constructed from the Kirkwood estimate of the diffusivity [8]

$$
\frac{1}{R_{\mathrm{H}}} \sim D_{\mathrm{K}}=\frac{1}{4}\left(\frac{1}{N}+\sqrt{\pi} h^{*} \frac{1}{R_{\mathrm{I}}}\right)
$$

where, $R_{\mathrm{I}}$ is the inverse radius (or sometimes known as the “dynamic" radius):

$$
\frac{1}{R_{\mathrm{I}}} \equiv \frac{1}{N^{2}} \sum_{\substack{\mu, \nu=1 \\ \mu \neq \nu}}^{N}\left\langle\frac{1}{r_{\mu \nu}}\right\rangle .
$$

Here, $r_{\mu \nu}$ is the distance between the beads $\mu$ and $\nu$. It is observed from experiments that $\alpha_{\mathrm{H}}<\alpha_{\mathrm{g}}$, and for the typical maximum molecular weights studied, the effective scaling exponent (defined as $\nu^{\text {eff }}=1 / 2+\partial \log \alpha / \partial \log M$ ) is $\nu^{\text {eff }}=0.59$ for $\alpha_{\mathrm{g}}$ and $\nu^{\text {eff }}=0.57$ for $\alpha_{\mathrm{H}}$. An early explanation of this anomalous dynamic scaling behavior employed a model of self-excluding Gaussian blobs [4] to show that the segments within the Gaussian blob have more influence on $1 / R_{\mathrm{I}}$ than on $R_{\mathrm{g}}$. It was shown that this leads to a swelling in $R_{\mathrm{I}}$, defined as $\alpha_{\mathrm{I}}=R_{\mathrm{I}} / R_{\mathrm{I}}^{\theta}$, which obeys $\alpha_{\mathrm{I}}<\alpha_{\mathrm{g}}$. The effective exponent for $R_{\mathrm{I}}$ was shown to reach the asymptotic value of $\nu=0.59$ much more slowly than that for $R_{\mathrm{g}}$, and an approximate estimate provided $\nu^{\mathrm{eff}}=0.57$ for $R_{\mathrm{I}}$ for the experimental system considered. This idea and other modified blob theories have subsequently been discounted by Monte Carlo simulations [9, 10] of excluded volume chains which show that the model ignores the excess swelling of internal parts of the chain and that in fact the effective exponent is significantly closer to 0.59 than 0.57 [11], a fact supported by renormalisation group (RG) calculations [3] and our own results shown here.

The crossover scaling behaviour (from the $\theta$-limit to the good-solvent limit) of static properties, such as $\alpha_{\mathrm{g}}$ is a well studied problem. One of the most successful and widely used approaches is the Edwards continuous chain $(N \rightarrow \infty)$ representation of the bead-spring model [7]. RG calculations have shown that this model correctly captures the static scaling behavior for temperatures asymptotically near the $\theta$ temperature, in close agreement with experimental observations [3, 11, 12, 13]. However, RG calculations [14] of the crossover of dynamic swelling ratios based on the definition of the Kirkwood diffusivity lead to $\alpha_{\mathrm{I}} \approx \alpha_{\mathrm{g}}$ (to within $1 \%$ ).

An alternative explanation for the lower value of $\alpha_{\mathrm{I}}$ is the existence of a draining effect in hydrodynamic interactions [5]. This implies the introduction of another (draining) parameter into the theory. However, a recent compilation of dynamic data [15] shows a significant degree of universality in 
the crossover in $z$, clearly indicating the absence of another parameter (or that the chains are non-free draining).

In this work, we have reexamined the dynamic crossover problem, (restricting our attention to the Edwards' continuum chain description) in the light of three recent developments. First is the introduction of a scheme to simulate the continuum Edwards model using the Brownian dynamics (BD) method [16], which permits the study of dynamical and rheological properties. In this scheme, a repulsive Gaussian EV potential is used to mimic the $\delta$-function potential in the Edwards model, and the mapping of the model parameters to the EV parameter $z$ is shown to occur in the continuous chain limit [17]. The second is the advance made in the treatment of fluctuating HI using fast approximation methods [18]. The third is the resolution of a long standing problem with regard to the diffusivity of a polymer chain. Liu and Dünweg [19] have numerically proven that for chains with fluctuating HI, the long time diffusivity is lower than the short time Kirkwood estimate $D_{\mathrm{K}}$, and is given by $D=D_{\mathrm{K}}-D_{1}$, where $D_{1}$ is the equilibrium time correlation function of the drift term in the stochastic differential equation governing the motion of the beads of the chain [20]. These developments permit us to compute the diffusivity from the Edwards model at a finite $z$ and obtain the crossover exactly in the non-free draining limit. We show that in the crossover regime $\alpha_{\mathrm{I}} \approx \alpha_{\mathrm{g}}$, and $\alpha_{\mathrm{H}} \mathrm{ob}-$ tained from the long-time diffusivity is able to reproduce the observed anomaly in the swelling of hydrodynamic radius.

To simulate the continuum Edwards model, we begin with a discrete bead-spring chain with $N$ beads subject to Brownian motion. The dimensionless displacement of a bead position $\Delta R_{\mu i}$ during a finite time increment $\Delta t$ is taken to be [21]:

$$
\Delta R_{\mu i}=\frac{1}{4} D_{\mu \nu i j} F_{\nu j} \Delta t+\frac{1}{\sqrt{2}} B_{\mu \nu i j} \Delta W_{\nu j},
$$

where, the summation convention is implied for repeated indices. and a Greek subscript $\mu, \nu, \ldots=[1,2, \ldots, N]$ denotes the bead index and a Roman subscript $i, j, \ldots=[1,2,3]$ denotes a Cartesian coordinate. Here, $D_{\mu \nu i j}$ is the diffusion tensor, $F_{\mu i}$ is a matrix of the total body force acting on the beads, $B_{\mu \nu i j}$ is taken as the square root matrix of $D_{\mu \nu i j}$ defined as $B_{\mu \theta i k} B_{\nu \theta j k} \equiv D_{\mu \nu i j}$, and $\Delta W_{\mu i}$ is an increment to the Weiner process $W_{\nu j}$. In the absence of EV interactions, the distribution of bead connector vectors is assumed to be Gaussian. A repulsive narrow-Gaussian potential is used to regularise the $\delta$-function $\mathrm{EV}$ potential [17] considered in the Edwards model [7]. The potential is parameterised by $z^{*}$ representing the strength and $d^{*}$ representing its width [16]. The solvent quality (EV parameter) is given by $z=z^{*} \sqrt{N}$, obtained in the simultaneous limit of $N \rightarrow \infty$ and $z^{*} \rightarrow 0$. In Ref. [16] the expression $d^{*}=K z^{* 1 / 5}$ (where $K$ is a constant) was found to be a computationally efficient way to reach the limit $d^{*} \rightarrow 0$, which recovers the $\delta$-function. This method was shown to accurately predict the swelling of the gyration radius [16] observed in experiments [22]. To simulate hydrodynamic interactions (HI) between the beads we have used the usual Rotne-Prager-Yamakawa expression for the HI tensor, and an accelarated method of finding the square root matrix $B_{\mu \nu i j}$ [18]. These and other details of the simulation are discussed elsewhere [21].

Fixman [20] established from a preaveraged treatment for Gaussian chains that dynamic correlations play a minor role in the diffusivity of chains. He showed that the long time diffusivity is given by $D=D_{\mathrm{K}}-D_{1}$, where the contribution $D_{1}>0$ is related to the time-correlation function of the drift term in Equation (3), and is only about $2 \%$ of $D_{\mathrm{K}}$. Defining $A_{i}=\left(\sum_{\mu} D_{\mu \nu i j} F_{\nu j}\right) / 4$, we have

$$
D_{1}=\frac{1}{3 N^{2}} \int_{0}^{\infty} \mathrm{d} t\left\langle A_{i}(0) A_{i}(t)\right\rangle .
$$

Liu and Dünweg [19], who recently performed simulations to obtain diffusivity $D$ directly, and through the Fixman's relationship, effectively proved the relation by showing numerically (for excluded volume chains) the variation of diffusivity from its short time value $D_{\mathrm{K}}$ to the long time limit. Even in this case, it was found that the contribution of $D_{1}$ is only a small fraction of $D$. This is one of the prime reasons for the neglect of dynamic correlations in the diffusivity and the use instead, of the Kirkwood estimate $D_{\mathrm{K}}$-an approximation which we show below has gone unnoticed in attempts to explain dynamic scaling.

We have validated Fixman's expression in $\theta$-conditions with fluctuating HI, by carrying out similar simulations to that in Ref. [19]. In this work, we have used the computationally easier method of evaluating $D$, which is to evaluate the static average $D_{\mathrm{K}}$ and obtain $D_{1}$ from the time-correlation function. This immediately enables one to study the crossover of both the static estimate and the dynamically correlated value.

We define two swelling ratios for the two different size measures obtained from the diffusivity. The commonly used Kirkwood estimate gives the swelling of the inverse radius $\alpha_{\mathrm{I}}=R_{\mathrm{I}} / R_{\mathrm{I}}^{\theta}=D_{\mathrm{K}}^{\theta} / D_{\mathrm{K}}$, in which the second equation is obtained in the limit $N \rightarrow \infty$ (which is true for the continuum model). The hydrodynamic radius obtained from the long-time diffusivity has a swelling ratio $\alpha_{\mathrm{H}}=D^{\theta} / D . \alpha_{\mathrm{I}}$ is obtained accurately from free-draining runs by carrying out simulations at two different values of $K=1.0$ and 1.5. $\alpha_{\mathrm{H}}$ is obtained from separate runs incorporating fluctuating $\mathrm{HI}$ and is carried out for two values of HI parameter (which is the dimensionless radius of a bead), $h^{*}=0.19$ and 0.30 . The results from simulations performed at finite $N$ are extrapolated to $N \rightarrow \infty$, keeping $z$ and $h^{*}$ constant. This limit corresponds to the continuum Edwards model in the non-free draining limit $\left(h^{*} \sqrt{N} \rightarrow \infty\right)$.

The universal nature of the swelling ratios $\alpha_{\mathrm{I}}$ and $\alpha_{\mathrm{H}}$ is seen in Figure 1 The independence of the extrapolated value of $\alpha_{\mathrm{I}}$ from the width of the Gaussian repulsive potential indicates $z$ is the only relavant parameter. A similar behaviour is observed for $\alpha_{\mathrm{H}}$ (not shown here). To calculate $\alpha_{\mathrm{H}}$ we find it 

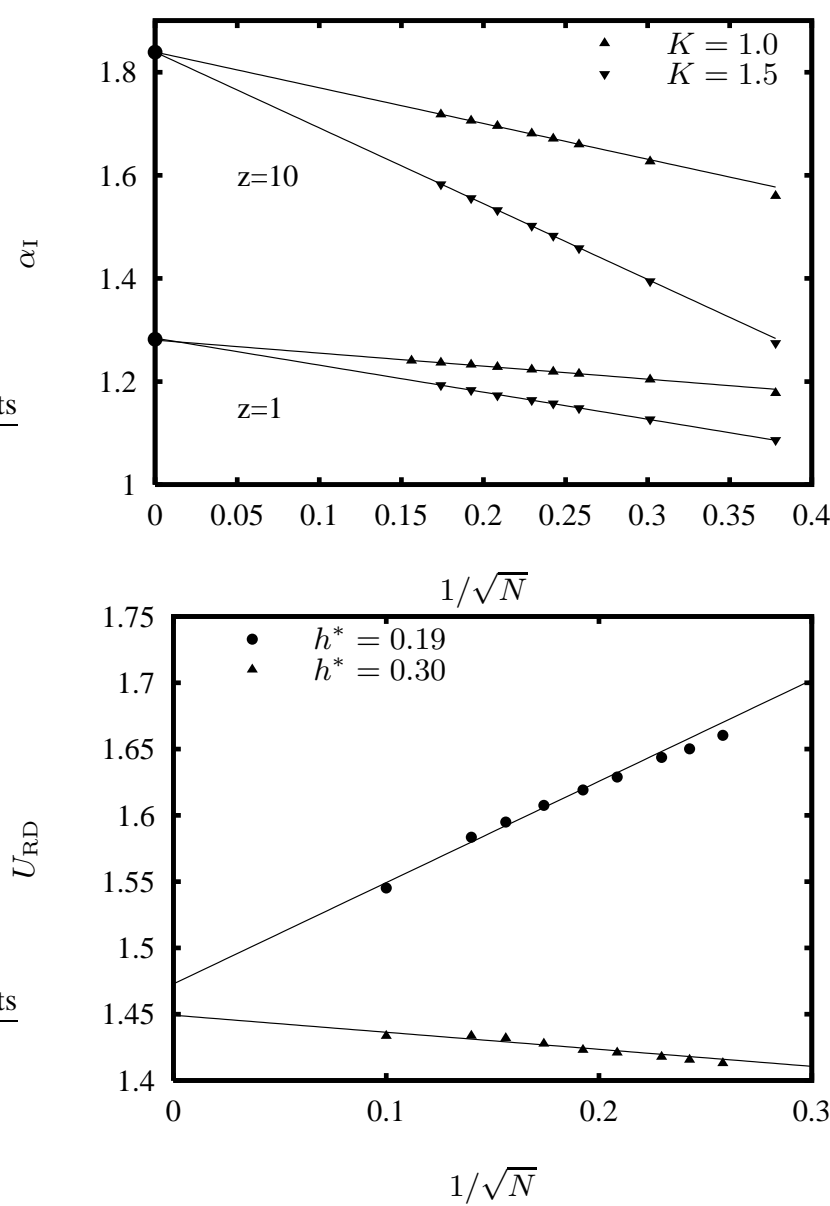

FIG. 1: Universality at a fixed solvent quality $z$ in the continuous chain limit $N \rightarrow \infty$. (a) The independence of the extrapolated value of $\alpha_{\mathrm{I}}$ from the width of the Gaussian repulsive potential and (b) A similar independence of $U_{\mathrm{RD}}$ from the HI parameter $h^{*}$ at a solvent quality $z=1$.

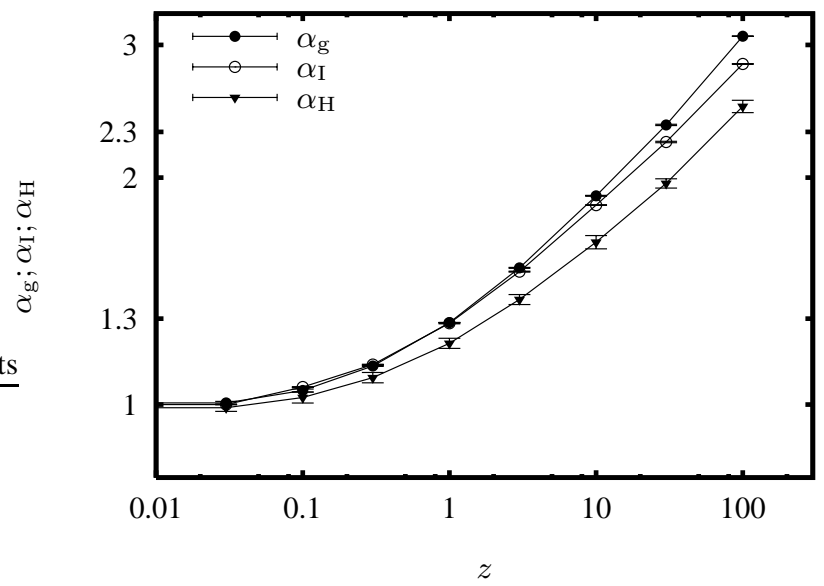

FIG. 2: Universal solvent quality crossover of swelling of two measures of polymer size based on diffusivity (inverse radius $\alpha_{\mathrm{I}}$ and hydrodynamic radius $\alpha_{\mathrm{H}}$ ) compared with the crossover of swelling of the radius of gyration $\left(\alpha_{\mathrm{g}}\right)$. convenient to use the following expression

$$
\alpha_{\mathrm{H}}=\frac{R_{\mathrm{H}}}{R_{\mathrm{g}}} \frac{R_{\mathrm{g}}}{R_{\mathrm{g}}^{\theta}} \frac{R_{\mathrm{g}}^{\theta}}{R_{\mathrm{H}}^{\theta}}=U_{\mathrm{RD}}^{-1} \alpha_{\mathrm{g}} U_{\mathrm{RD}}^{\theta}
$$

where, $U_{\mathrm{RD}} \equiv R_{\mathrm{g}} / R_{\mathrm{H}}$. This separates out the weak dependence of $\alpha_{\mathrm{H}}$ on $h^{*}$ at finite $N$ into the two strong dependences of each of $U_{\mathrm{RD}}$ and $U_{\mathrm{RD}}^{\theta} \cdot \alpha_{\mathrm{g}}$ is independent of $h^{*}$ and can be obtained accurately from free-draining simulations along with $\alpha_{\mathrm{I}}$. Our simulations of Gaussian chains with fluctuating HI yield a value of $U_{\mathrm{RD}}^{\theta}=1.38 \pm 0.01 . \alpha_{\mathrm{g}}$ is determined from free-draining simulations [16]. $U_{\mathrm{RD}}$ is determined by carrying out simulations at constant $h^{*}$, and the extrapolations to $N \rightarrow \infty$ are carried out for two values of $h^{*}=0.19$ and 0.30 as shown in Figure $1 \mathrm{~b}$. The two extrapolations can be seen to approach a common limiting value, which are not as close to each other as observed for the EV parameter. We believe that this is a limitation of the maximum $N$ that we have considered in the extrapolation, and a higher $N$ (which is difficult to simulate presently) should lower the margin of difference in the extrapolated value. In the results to follow, we indicate this margin through an error-bar in the data points.

The complete crossover of the two swelling ratios is shown in Figure 2 and is compared with that of $\alpha_{\mathrm{g}}$. It is observed that $\alpha_{\mathrm{I}} \approx \alpha_{\mathrm{g}}$ within error bounds for a large range of $z$, i.e., the cross-over of static size measures is nearly identical. This is a new result, calculated exactly for the first time for the continuum Edwards model. This is in contrast to the simple scaling arguments of the thermal blob model [4]. It is clear that the "spatial-crossover" explanation of the blob model is insufficient to show the observed difference in the crossover of the swelling of hydrodynamic radius. On the other hand, we see from Figure 2 that the crossover obtained from the long-time diffusivity is significantly different and $\alpha_{\mathrm{H}}<\alpha_{\mathrm{g}}$ for all values of $z$. Recognition of this fact is important since most of the theoretical calculations are based on the static size measure $\left(D_{\mathrm{K}}\right)$ ignoring dynamic correlations $\left(D_{1}\right)$.

The theoretical crossover behaviour can be compared with experiments in two ways. In one method we can compare the $z$-crossover of the swelling ratios, for which it is required to estimate the phenomenological parameter $z$ for the experimental system, which has a chemistry dependent prefactor. In the alternative approach, we can directly compare with two experimentally measured quantities, without the need to estimate $z$. We have chosen the most recent data (to our knowledge) on the hydrodynamic radius, collated in Ref. [15], in terms of a solvent quality they denote as $\tilde{z}$. We found that by taking $z=0.85 \tilde{z}$, we can shift the experimental data for the swelling of radius of gyration $\alpha_{\mathrm{g}}$ on to our theoretical curve. We then compare the crossover of $\alpha_{\mathrm{H}}$ using the same shift factor. Figure 3 shows this comparison leads to remarkable agreement. It is also observed that the crossover of $\alpha_{\mathrm{I}}$ lies outside the region of experimental accuracy. This provides a substantial evidence that dynamic correlations are important to describe the observed crossover behavior. We have also made a parameter-free comparison with the experimental data, 


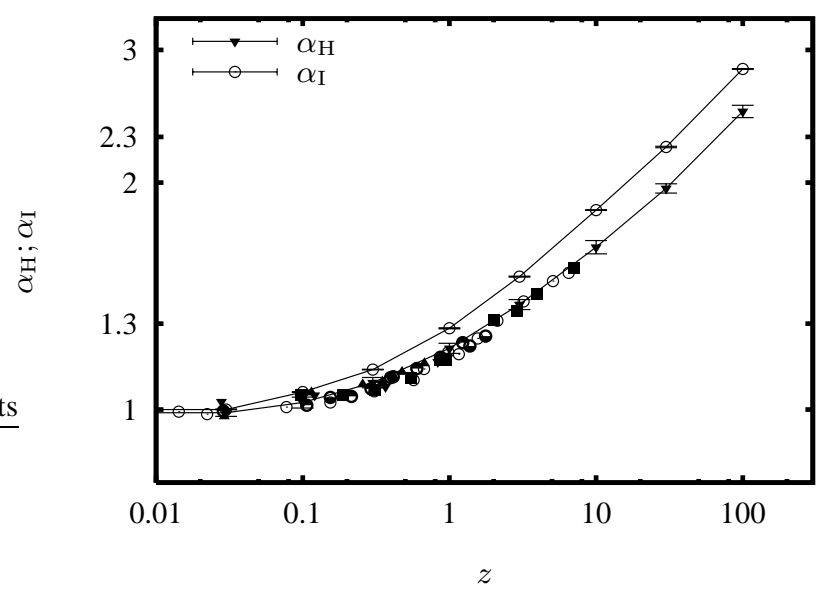

FIG. 3: Crossover of hydrodynamic radius: Comparison of theoretical swelling of static and dynamic measures (line with points) with experimental data (points) collated in Ref. [15].

as shown in Figure 4 for the measured values of $\alpha_{\mathrm{H}}$ against that of $\alpha_{\mathrm{g}}$, leading us to the same conclusion.

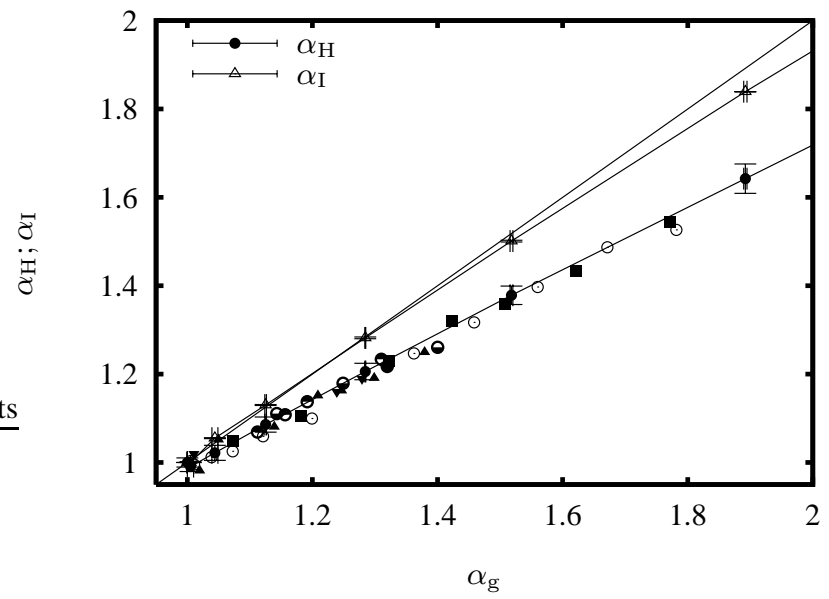

FIG. 4: Parameter free comparison with measured values of $\alpha_{\mathrm{H}}$ against $\alpha_{\mathrm{g}}$. Experiments are several data collated in Ref. [15].

Based on the limited set of points shown in Figure 2 effective exponents for the static and dynamic properties can be estimated at $z=10$, which is typically the largest experimental value of $z$. Taking $z \propto \sqrt{M}$, we find $R_{\mathrm{g}} \sim M^{0.59}$, $R_{\mathrm{I}} \sim M^{0.58}$, and $R_{\mathrm{H}} \sim M^{0.57}$. These results should be taken only as indicative values of the exponent near $z=10$, and are not conclusive critical exponents.

To summarize, Brownian Dynamics simulations of the continuum Edwards model were used to study the crossover behavior of the hydrodynamic radius of a polymer chain. We found that the commonly used static measure of diffusivity (obtained from the inverse radius $R_{\mathrm{I}}$ ) has nearly identical crossover to that of the gyration radius. It is required to consider the long time diffusivity (which has dynamic correlations) to explain the experimentally observed slow crossover of dynamic properties. Analytical calculations would be helpful to obtain further insight into the results obtained here by simulations.

This work has been supported by a grant from the Australian Research Council, under the Discovery-Projects program. The computations were carried out in the facilities provided by the Australian Partnership for Advanced Computation (APAC), and we are grateful for their support.

* Electronic address: P.Sunthar@gmail.com

$\dagger$ Electronic address: Ravi.Jagadeeshan@eng.monash.edu.au

[1] P.-G. de Gennes, Scaling concepts in polymer physics (Cornell University Press, Ithaca, 1979).

[2] W. W. Graessley, R. C. Hayward, and G. S. Grest, Macromolecules 32, 3510 (1999).

[3] J. F. Douglas and K. F. Freed, Macromolecules 17, 2344 (1984), http://dx.doi.org/10.1021/ma00141a026

[4] G. Weill and J. des Cloizeaux, J. Phys. (France) 40, 99 (1979).

[5] J. F. Douglas and K. F. Freed, Macromolecules 17, 2354 (1984), http://dx.doi.org/10.1021/ma00141a027

[6] M. Benmouna and A. Z. Akcasu, Macromolecules 11, 1187 (1978), http://dx.doi.org/10.1021/ma60066a023

[7] S. F. Edwards, Proc. Phys. Soc. 85, 613 (1965).

[8] J. G. Kirkwood, J. Polym. Sci. 12, 1 (1954), http://dx.doi.org/10.1002/pol.1954.120120102

[9] L. Schäfer and A. Baumgärtner, J. Phys. (Paris) 47, 1431 (1986).

[10] A. J. C. Ladd and D. Frenkel, Macromolecules 25, 3435 (1992), http://dx.doi.org/10.1021/ma00039a019

[11] L. Schäfer, Excluded Volume Effects in Polymer Solutions (Springer-Verlag, Berlin, 1999).

[12] M. Muthukumar and B. G. Nickel, J. Chem. Phys. 86, 460 (1987), http://dx.doi.org/10.1063/1.452586

[13] J. des Cloizeaux, R. Conte, and G. Jannink, J. Phys. Lett. (Paris) 46, L 595 (1985).

[14] J. F. Douglas and K. F. Freed, Macromolecules 18, 201 (1985), http://dx.doi.org/10.1021/ma00144a017

[15] Y. Tominaga, I. Suda, M. Osa, T. Yoshizaki, and H. Yamakawa, Macromolecules 35, 1381 (2002), http://dx.doi.org/10.1021/ma011348x

[16] K. S. Kumar and J. R. Prakash, Macromolecules 36, 7842 (2003), http://dx.doi.org/10.1021/ma034296f

[17] J. R. Prakash, Chem. Eng. Sci. 56, 5555 (2001), http://dx.doi.org/10.1016/S0009-2509(01)00154-3

[18] R. M. Jendrejack, M. D. Graham, and J. J. de Pablo, J. Chem. Phys. 113, 2894 (2000), http://dx.doi.org/10.1063/1.1305884

[19] B. Liu and B. Dünweg, J. Chem. Phys. 118, 8061 (2003), http://dx.doi.org/10.1063/1.1564047

[20] M. Fixman, Macromolecules 14, 1710 (1981), http://dx.doi.org/10.1021/ma50007a019

[21] P. Sunthar and J. R. Prakash, Macromolecules 38, 617 (2005), http://dx.doi.org/10.1021/ma0359411

[22] Y. Miyaki and H. Fujita, Macromolecules 14, 742 (1981), http://dx.doi.org/10.1021/ma50004a053 